\title{
Some Problematic Aspects of Coliform Bacteria Clustering on Medical Images in the Task of Identifying Possible Diseases
}

\author{
Vyacheslav Lyashenko ${ }^{1}$, Pavel Orobinsky ${ }^{1}$ \\ lyashenko.vyacheslav@gmail.com \\ ${ }^{1}$ Department of Informatics, Kharkiv National University of RadioElectronics, Ukraine \\ Received: December 16, 2020 \\ Received in revised: January 2, 2021 \\ Accepted: January 6, 2020
}

\begin{abstract}
Medical image analysis methods are one of the sources for obtaining additional information about the investigated phenomena. We are looking at images of coliform bacteria. Analysis of these images allows you to determine the possibility of developing certain diseases. To do this, it is necessary to cluster the set of bacteria and count the bacteria. The paper highlights the features of clustering for coliform bacteria. Clustering results for real data are presented.
\end{abstract}

Keywords: Clustering, Coliform Bacteria, Medical Images, Image Processing

\section{Introduction}

A medical image is an image of cells, tissue sections of various human organs. These images are usually taken under a microscope. Thus, the medical image is the image of the microworld. These images help visualize and understand the processes that are hidden to the human eye (Karthick \& Maniraj, 2019; Lyashenko, Babker \& Kobylin, 2016; Orobinskyi, Petrenko \& Lyashenko, 2019). It can also be processes in those places of the human body that are inaccessible. Medical images are data that have their own specific properties and features of their use (Orobinskyi, Petrenko \& Lyashenko, 2019; Lyashenko, Sinelnikova, Zeleniy \& Babker, 2020; Babker \& Lyashenko, 2020). Therefore, medical images are a source of information for decision-making in the diagnosis and treatment of various diseases.

One example of medical imaging is coliform bacteria. Coliform bacteria are a collection of bacteria that are often used as indicators of the sanitary quality of food and water (Jeon \& et al., 2019; Wang \& Deng, 2019). At the same time, coliform bacteria indicate the possibility of the presence of other pathogenic organisms. It is important to know the quantitative characteristics of the accumulation of such bacteria. It is the concentration of bacteria that helps to determine the possibility of infection, to correctly diagnose the alleged disease.

Various image processing methods are used to analyze medical images (Lyashenko, Sinelnikova, Zeleniy \& Babker, 2020; Rabotiahov, Kobylin, Dudar \& Lyashenko, 2018; Lyashenko, Babker $\&$ Kobylin, 2016). These methods can be different, and their use depends on the problem that needs to be solved in the process of the corresponding analysis. Therefore, it is important not only to select certain methods for image analysis, but also to justify the sequence of their use for such data analysis. Some of these issues are discussed in this paper. 


\section{Brief overview of related research papers}

The analysis and processing of medical images is one of the areas of research that is developing in various directions and has many relevant publications.

M. I. Razzak, S. Naz and A. Zaib discuss various problematic aspects in medical imaging (Razzak, Naz \& Zaib, 2018). At the same time, attention is paid to the fact that each medical image is unique and requires an individual approach in each case. The authors also note the importance of such a procedure as the analysis of medical images in the process of diagnosing diseases. At the same time, the authors pay special attention to the analysis of medical images based on neural networks.

The study by authors D. Shen, G. Wu, and H. I. Suk provides a general overview of image analysis methods in the field of medical imaging (Shen, Wu \& Suk, 2017). Particular attention is paid to the method of neural networks, which are widely used in the processing of medical images. Nevertheless, the authors of the studies emphasize the need to preserve the original information as a result of using image analysis methods.

A review of medical image processing methods is presented in (Goel, Yadav \& Singh, 2016). The study authors note that medical imaging is becoming an important procedure in various medical applications. This procedure provides additional information. This additional information helps to improve the efficiency of diagnosis of various diseases and improve the treatment process. The authors also highlight certain problematic aspects in the processing of medical images.

R. Merjulah and J. Chandra in their work pay special attention to segmentation as one of the procedures for processing medical images (Merjulah \& Chandra, 2017). The authors emphasize that segmentation is one of the most common methods for processing medical images. In this case, the main purpose of segmentation is to highlight the area of interest for its subsequent processing and analysis. At the same time, it is necessary to pay attention to clustering, which allows us to analyze all the objects that interest us.

In work (Wang \& et al., 2020), the issues of detection and classification of living bacteria are considered. To do this, the authors use a sequence of several images and analysis of such images based on deep learning neural networks. It can also be seen from this work that an important aspect is the clustering of bacteria that can be distinguished in the image.

M. Hiremath discusses the procedure for segmentation and recognition of E.coli bacterial cells (Hiremath, 2019). M. Hiremath uses medical images taken with a microscope for appropriate analysis. At the same time, the author of the work speaks about the need for preliminary coloring of such images. Also an important element of image processing is filtering small particles.

In work (Cernicchiaro \& et al., 2019), the problem of quantitative determination of bacteria is considered to determine pollution and possible contamination of the environment.

D. Połap and M. Woźniak solve the problem of classifying different forms of bacteria (Połap \& Woźniak, 2019). To do this, the authors use a preliminary separation of different bacteria based on their descriptive characteristics. Next, a convolutional neural network model is used to flesh out this preliminary separation of bacteria. 
B. Kis, M. Unay, G. D. Ekimci, U. K. Ercan, and A. Akan use imaging techniques to count bacterial colonies (Kis \& et al., 2019). For this, various methods of image analysis are used. At the same time, one of the problematic issues is the count of bacteria in each colony.

Thus, we can say that there are a number of problematic aspects that can be encountered as a result of the application of processing methods for the analysis of coliform bacteria images.

Some examples of coliform bacteria images in the context of problematic aspects of their processing

Figure 1 shows various images of coliform bacteria (Orobinskyi, Deineko \& Lyashenko, 2020).

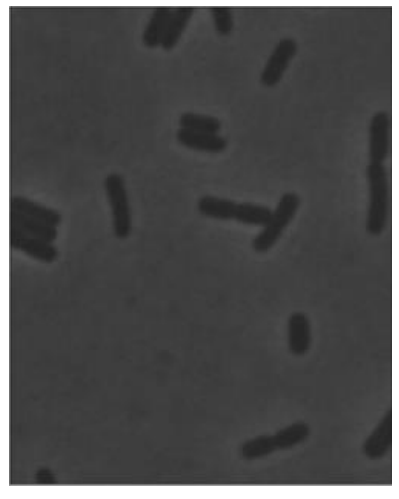

a)

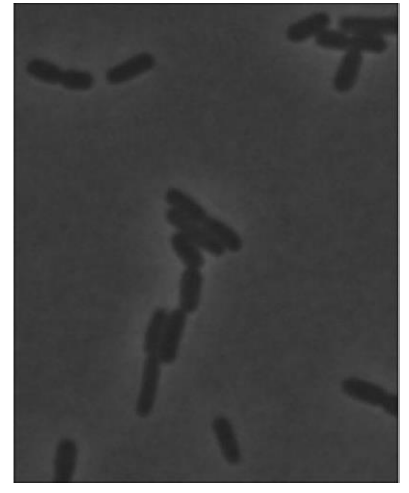

b)

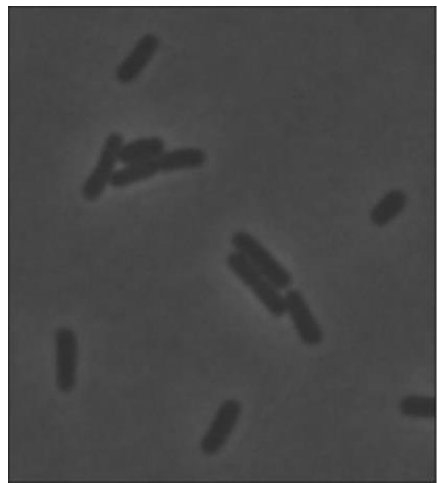

c)

Figure 1. Various images of coliform bacteria

We see various clumps of coliform bacteria. At the same time, some clusters make it possible to distinguish between single bacteria, but for some clusters this is difficult to do. Individual fragments of such clusters are shown in Figure 2.

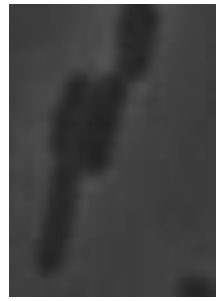

a)

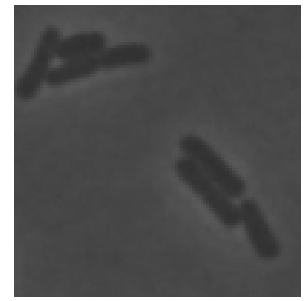

b)

Figure 2. Fragments of bacteria clusters that are difficult to separate into individual bacteria

The difficulty in separating clusters of bacteria into individual bacteria is due to the fact that it is difficult to determine the junction points between such bacteria (Figure 3). In work (Orobinskyi \& Lyashenko, 2020) it is shown that the solution of this problem should be divided into two subtasks - finding the junction points of two objects and determining the boundaries of objects. 


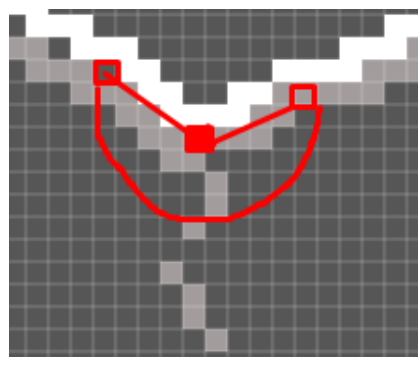

a)

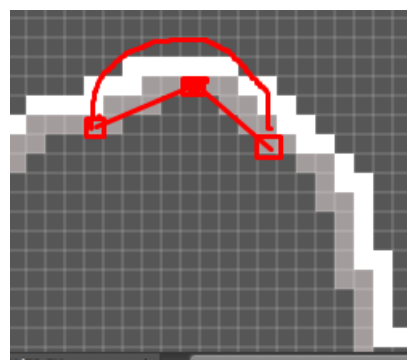

b)

Figure 3. Joint points: a) is a joint point, b) is not a joint point

Thus, one of the problematic aspects in the clustering of accumulations of bacteria is the determination of the boundary points. The solution to this problem is to find the optimal relationship between the individual boundary points of the images of bacteria. This can be done using algorithms for finding the optimal path on the graph (Orobinskyi \& Lyashenko, 2020).

Another problematic aspect when clustering bacterial clumps is to improve the quality of the original image. The main issue in this context is not to degrade the quality of the original image (Orobinskyi, Deineko \& Lyashenko, 2020). Examples of such low-quality processing are shown in Figure 4, which shows the results of filtering the original image (Figure 1b).

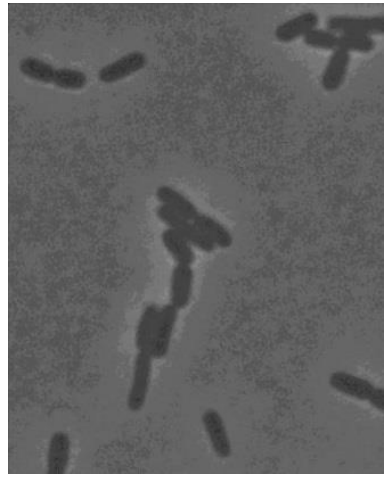

a)

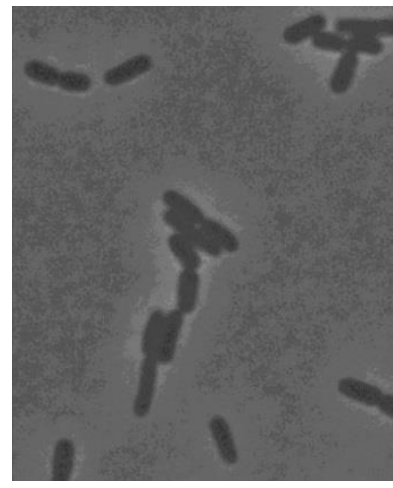

b)

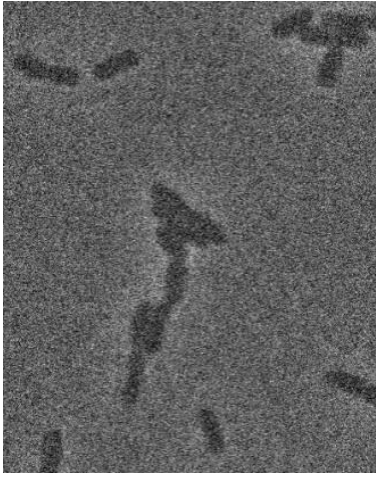

c)

Figure 4. Examples of different ways to filter the original image

Therefore, when solving the problem of clustering accumulations of bacteria in medical images, it is necessary to carefully approach the filtering procedure of the original image. At the same time, it is also necessary to take into account the geometric and morphological characteristics of bacterial clusters and their individual varieties.

\section{An example of clustering results bacteria concentrations}

First of all, we note that the problem of clustering a set of bacteria will be considered as the problem of coloring each individual bacterium with a certain color. This then makes it possible to quickly count the number of bacteria and determine a possible identification of the degree of disease. Figure 5 (Figure 5a - original image, Figure 5b-processed image) shows an example of clustering a set of bacteria in conditions when such a set is simple and does not have critical junctions between individual bacteria. 


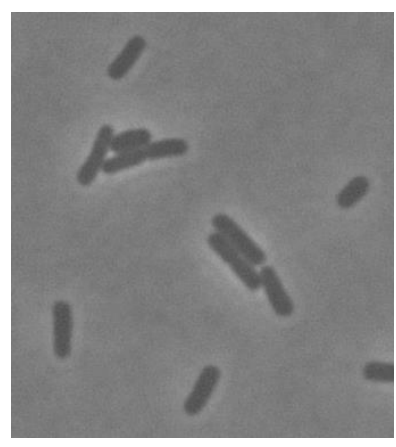

a)

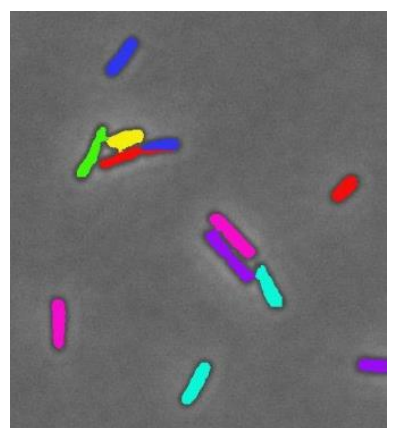

b)

Figure 5. Example of clustering a set of bacteria

We see that all bacteria have their own unique color. This allows for a simple and quick enumeration of bacteria in their entirety.

For clustering, we used: the possible minimum and maximum size of bacteria, the depth of separation of bacteria at the junction points, the size of the area of the local extremum, the geometry of the connectivity points in the vicinity of the junction points. These are the so-called clustering input parameters.

Figure 6 (Figure $6 \mathrm{a}$ - the original image, the rest of the images - the clustering results for different input parameters) shows an example of clustering a set of bacteria in difficult conditions: when the junction points are difficult to distinguish; when a filtering operation was performed that could not remove all false points.

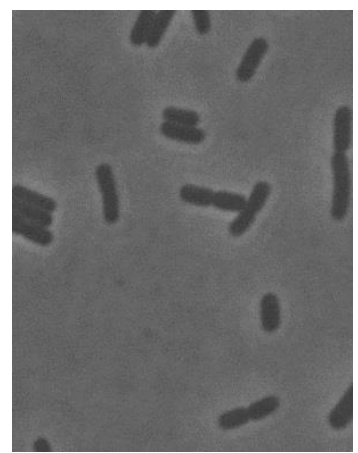

a)

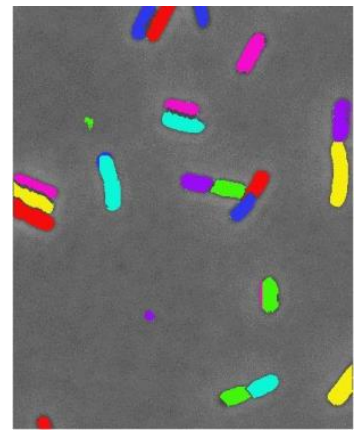

d)

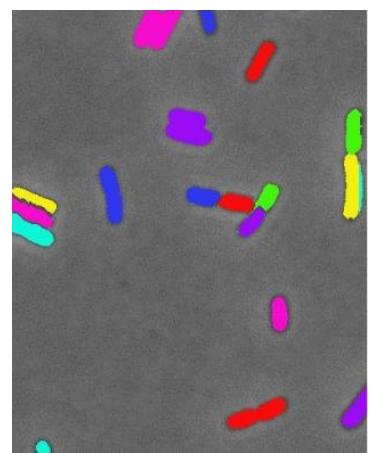

b)

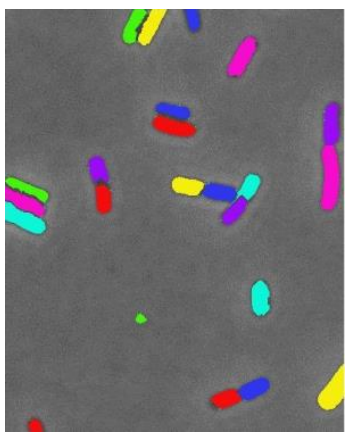

e)

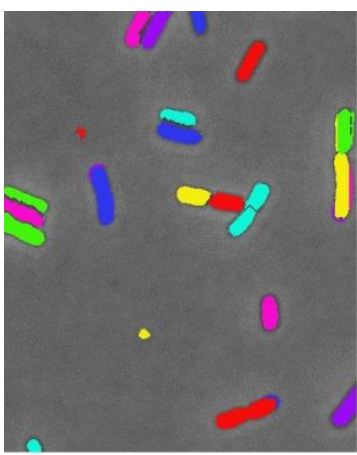

c)

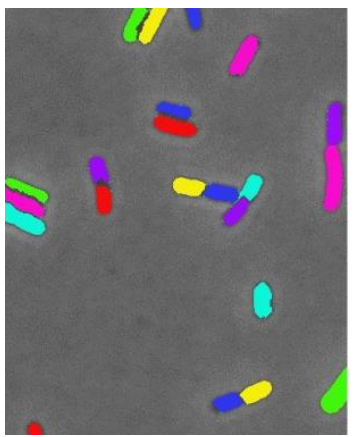

f)

Figure 6. Example of clustering a set of bacteria in difficult conditions 
We can see that the chosen clustering kit helps to separate all bacteria. At the same time, regardless of the parameters of the clustering set, we get a generally good result.

\section{Conclusion}

We analyzed medical imaging techniques for coliform bacteria. One of the most important procedures for such image processing is clustering a population of bacteria. This helps to count all bacteria and determine the level of possible disease. We also noted the problematic aspects of medical image analysis when the clustering procedure is applied. We have provided examples of clustering for real data. Also shown is a dataset that can be used to cluster a population of coliform bacteria.

\section{References}

Babker, A. M., \& Lyashenko, V. (2020). Wavelet Analysis as Research Tool Image Cytological Preparations. J Clin Exp Pathol, 10, 382.

Cernicchiaro, N., Oliveira, A. R., Hoehn, A., Cull, C. A., Noll, L. W., Shridhar, P. B., ... \& Sanderson, M. W. (2019). Quantification of bacteria indicative of fecal and environmental contamination from hides to carcasses. Foodborne Pathogens and Disease, 16(12), 844-855.

Goel, N., Yadav, A., \& Singh, B. M. (2016). Medical image processing: a review. In 2016 Second International Innovative Applications of Computational Intelligence on Power, Energy and Controls with their Impact on Humanity (CIPECH), 57-62.

Hiremath, M. (2019). Segmentation and Recognition of E. coli Bacteria Cell in Digital Microscopic Images Based on Enhanced Particle Filtering Framework. In Emerging Research in Computing, Information, Communication and Applications, 503-512.

Jeon, D. J., Ligaray, M., Kim, M., Kim, G., Lee, G., Pachepsky, Y. A., ... \& Cho, K. H. (2019). Evaluating the influence of climate change on the fate and transport of fecal coliform bacteria using the modified SWAT model. Science of the Total Environment, 658, 753762.

Karthick, S., \& Maniraj, S. (2019). Different Medical Image Registration Techniques: A Comparative Analysis. Current Medical Imaging, 15(10), 911-921.

Kis, B., Unay, M., Ekimci, G. D., Ercan, U. K., \& Akan, A. (2019). Counting Bacteria Colonies Based on Image Processing Methods. In 2019 Medical Technologies Congress (TIPTEKNO), 1-4.

Lyashenko, V. V., Babker, A. M., \& Kobylin, O. A. (2016). The methodology of wavelet analysis as a tool for cytology preparations image processing. Cukurova Medical Journal, 41(3), 453-463.

Lyashenko, V., Babker, A. M. A. A., \& Kobylin, O. (2016). Using the methodology of wavelet analysis for processing images of cytology preparations. National Journal of Medical Research, 6(1). 98-102.

Lyashenko, V., Sinelnikova, T., Zeleniy, O., \& Babker, A. M. A. (2020). Specifics of Using Image Processing Techniques for Blood Smear Analysis. Journal La Medihealtico, 1(6), 1-6. 
Merjulah, R., \& Chandra, J. (2017). Segmentation technique for medical image processing: A survey. In 2017 International Conference on Inventive Computing and Informatics (ICICI), 1055-1061.

Orobinskyi, P., \& Lyashenko, V. (2020). The Task of Identifying the Various Objects' Boundary Points of the Medical Image as the Optimal Path Search on the Graph. International Journal of Emerging Trends in Engineering Research, 8(3), 824-829.

Orobinskyi, P., Deineko, Z., \& Lyashenko, V. (2020). Comparative Characteristics of Filtration Methods in the Processing of Medical Images. American Journal of Engineering Research, 9(4), 20-25.

Orobinskyi, P., Petrenko, D., \& Lyashenko, V. (2019). Novel Approach to Computer-Aided Detection of Lung Nodules of Difficult Location with Use of Multifactorial Models and Deep Neural Networks. In 2019 IEEE 15th International Conference on the Experience of Designing and Application of CAD Systems (CADSM), 1-5.

Połap, D., \& Woźniak, M. (2019). Bacteria shape classification by the use of region covariance and convolutional neural network. In 2019 International Joint Conference on Neural Networks (IJCNN), 1-7.

Rabotiahov, A., Kobylin, O., Dudar, Z., \& Lyashenko, V. (2018). Bionic image segmentation of cytology samples method. In 2018 14th International Conference on Advanced Trends in Radioelecrtronics, Telecommunications and Computer Engineering (TCSET), 665670 .

Razzak, M. I., Naz, S., \& Zaib, A. (2018). Deep learning for medical image processing: Overview, challenges and the future. In Classification in BioApps, 323-350.

Shen, D., Wu, G., \& Suk, H. I. (2017). Deep learning in medical image analysis. Annual review of biomedical engineering, 19, 221-248.

Wang, H., Koydemir, H. C., Qiu, Y., Bai, B., Zhang, Y., Jin, Y., ... \& Ozcan, A. (2020). Earlydetection and classification of live bacteria using time-lapse coherent imaging and deep learning. arXiv preprint arXiv:2001.10695.

Wang, J., \& Deng, Z. (2019). Modeling and predicting fecal coliform bacteria levels in oyster harvest waters along Louisiana Gulf coast. Ecological Indicators, 101, 212-220.

Copyright (C 2021, Journal of Asian Multicultural Research for Educational Study, Under the license CC BY-SA 4.0 